\title{
Immunomodulatory effect of mesenchymal stem cells and mesenchymal stem-cell-derived exosomes for COVID-19 treatment
}

\author{
Kaavya Jayaramayya ${ }^{1,2}$, Iyer Mahalaxmi ${ }^{2}$, Mohana Devi Subramaniam ${ }^{3}$, Neethu Raj ${ }^{1}$, Ahmed Abdal Dayem ${ }^{4}$, \\ Kyung Min Lim ${ }^{4}$, Se Jong Kim ${ }^{4}$, Jong Yub An ${ }^{4}$, Yoonjoo Lee ${ }^{4}$, Yujin Choi ${ }^{4}$, Arthi Kirubhakaran ${ }^{1}$, Ssang-Goo Cho ${ }^{4, *} \mathcal{E}^{2}$ \\ Balachandar Vellingiri, ${ }^{1, *}$ \\ ${ }^{1}$ Human Molecular Cytogenetics and Stem Cell Laboratory, Department of Human Genetics and Molecular Biology, Bharathiar University, \\ Coimbatore 641-046, ${ }^{2}$ Department of Zoology, Avinashilingam Institute for Home Science and Higher Education for Women, Coimbatore \\ 641-043, ${ }^{3}$ SN ONGC Department of Genetics and Molecular Biology, Vision Research Foundation, Sankara Nethralaya campus, Chennai \\ 600-006, India, ${ }^{4}$ Department of Stem Cell and Regenerative Biotechnology, Konkuk University, Seoul 05029, Korea
}

\begin{abstract}
The world has witnessed unimaginable damage from the coronavirus disease-19 (COVID-19) pandemic. Because the pandemic is growing rapidly, it is important to consider diverse treatment options to effectively treat people worldwide. Since the immune system is at the hub of the infection, it is essential to regulate the dynamic balance in order to prevent the overexaggerated immune responses that subsequently result in multiorgan damage. The use of stem cells as treatment options has gained tremendous momentum in the past decade. The revolutionary measures in science have brought to the world mesenchymal stem cells (MSCs) and MSC-derived exosomes (MSC-Exo) as therapeutic opportunities for various diseases. The MSCs and MSCExos have immunomodulatory functions; they can be used as therapy to strike a balance in the immune cells of patients with COVID-19. In this review, we discuss the basics of the cytokine storm in COVID-19, MSCs, and MSC-derived exosomes and the potential and stem-cell-based ongoing clinical trials for COVID-19. [BMB Reports 2020; 53(8): 400-412]
\end{abstract}

\section{INTRODUCTION}

The world has been facing a dreadful situation due to the spread of the Severe Acute Respiratory Syndrome-Coronavirus-2

*Corresponding authors. Balachandar Vellingiri, Tel: +91-422-2422514, +91-422-2422222; Fax: +91-422-2422387; E-mail: geneticbala@buc. edu.in, geneticbala@yahoo.co.in; Ssang-Goo Cho, Tel: +82-2-4504207; Fax: +82-2-444-4207; E-mail: ssangoo@konkuk.ac.kr, ssangoo33@ gmail.com

https://doi.org/10.5483/BMBRep.2020.53.8.121

Received 5 June 2020

Keywords: COVID-19, Exosome, Immunomodulation, Mesenchymal stem cells (MSCs), Ongoing Clinical trials, Therapy
(SARS-CoV-2) (1). However, neither confirmed effective antiviral medications nor vaccines are available to deal with this emergency (2). Many reports have suggested that it is the cytokine storm in COVID-19 that leads to acute respiratory distress syndrome (ARDS) (3). The cytokine storm in COVID-19 refers to the fact that a variety of cytokines are rapidly produced after viral infections (4). In addition, such a cytokine storm induces hypoxia, and direct viral infection can cause cellular damage. Multiorgan damage and injury have been concomitant with COVID-19, and can be observed more in patients with a more severe form of the disease (5).

Stem cells are specialized cells that can renew themselves by means of cell division and can differentiate into multilineage cells. Mesenchymal stem cell (MSCs) have immunomodulatory features and secrete cytokines and immune receptors that regulate the microenvironment in the host tissue (6). In addition, it has been observed that the crucial role of MSCs in therapy has been mediated by exosomes released by the MSCs. These exosomes have exhibited immunomodulatory, antiviral, anti-fibrotic, and tissue-repair-related functions in vivo; similar effects have been observed in vitro (6).

\section{COVID-19 AND THE IMMUNE SYSTEM}

The dynamic equilibrium maintained by innate and adaptive immunity is essential for impeding the progression of COVID-19 (7). In patients infected with SARS-CoV-2, the plasma levels of IL-1 $\beta$, IL-1RA, IL-7, IL-8, IL-10, IFN- $\gamma$, monocyte chemoattractant peptide (MCP)-1, macrophage inflammatory protein (MIP)-1A, MIP-1B, G-CSF, and TNF- $\alpha$ are significantly higher than in controls. The levels of these factors are also increased in patients who were admitted to ICUs (8). Similarly, reductions in the levels of T cells and NK cells have been observed in COVID-19 patients (9). The loss of such cells can impair the immune system (10). The levels of the helper T cells, cytotoxic suppressive T cells, and regulatory $\mathrm{T}$ cells are much lower in

ISSN: 1976-670X (electronic edition)

Copyright (c) 2020 by the The Korean Society for Biochemistry and Molecular Biology

(c) This is an open-access article distributed under the terms of the Creative Commons Attribution Non-Commercial License (http://creativecommons.org/licenses/by-nc/4.0) which permits unrestricted non-commercial use, distribution, and reproduction in any medium, provided the original work is properly cited. 
patients with COVID-19 than in their healthy and less severe counterparts. The decrease in the regulatory $T$ cells may hamper their ability to inhibit the chronic inflammation (11). Interestingly, a remarkable increase is observed in the naïve T cells, where as the memory $T$ cells are reduced in infected patients (10). The reduced expression of memory cells may be a plausible explanation for the increased rates of reinfection by SARSCoV-2.

\section{THE CYTOKINE STORM}

SARS-CoV-2 binds to the Angiotensin-converting enzyme 2 (ACE2) receptor and enters the host cell (1). During infection, the innate and adaptive immune systems work together to inactivate the virus. Since leukocytes and neutrophils are present in higher concentrations in COVID-19 individuals, these immune cells may result in the cytokine storm (10). After viral entry, the virus induces pyroptosis and cell death. The dead cells recruit macrophages to the site of injury that phagocytose them. The phagocytes then express damage-associated molecular patterns (DAMPs), which bind to the toll-like receptors (TLR) and induce nuclear factor kappa $\mathrm{B}(\mathrm{NF}-\kappa \beta)$ signalling by means of the MyD88 pathway. NF- $\kappa \beta$ enters the nucleus and catalyzes the transcription of pro-IL-1 $\beta$ and procaspase-1. When additional signals are detected, the pro-IL-1 $\beta$ and procaspase 1 are cleaved into IL-1 $\beta$ and caspase 1 (12). The activated NOD-, LRR- and pyrin domain-containing protein 3 (NLRP3) recruits the apoptosis-associated speck-like protein containing a caspase recruitment domain (ASC) and pro-caspase- 1 to form the NLRP3 inflammasome (13). In addition, the phagocytosis releases ATP, which binds to the P2X purinoceptor 7 (P2RX7) and activates the inflammasome (14). The increased calcium levels caused by the viral proteins results in lysosomal damage, thereby releasing cathepsins that activate the inflammasome (15). Further, the binding of SARS-CoV-2 to the ACE2 reduces the available ACE2 receptors on the cell surface. This increases the levels of Angiotensin II (Angll) in the extracellular space, because ACE2 converts Angl and Angll into Ang 1-9 and Ang1-7, respectively. Angll increases the levels of TNF- $\alpha$ and IL- 6 in the cell that upregulates NF- $\kappa \beta$, activating the inflammasome (12). The continuous activation of the inflammasome results in a cytokine storm, which recruits more immune cells, necrosis, and cell death. This inflammasome pathway further causes tissue injury in various organs (Fig. 1).

\section{MSCs AND IMMUNOMODULATION}

MSCs are predominantly isolated from the bone marrow, adipose tissue, dental pulp, umbilical cord, Wharton's jelly, placenta, synovial fluid, endometrium, and peripheral blood. These cells exhibit different cell-surface markers and can be used for a variety of treatment options (Table 1). MSCs can undergo in vitro amplification and self-renewal, and have low immunogenicity and immune-modulatory functions; the latter have attracted attention in clinical trials (16). MSCs have been widely used in various cellular therapies, such as pre-clinical studies, as well as in some clinical trials, because of their high safety and efficacy $(17,18)$. MSCs can exert immune-modulatory effects in the host cells of both the innate and the
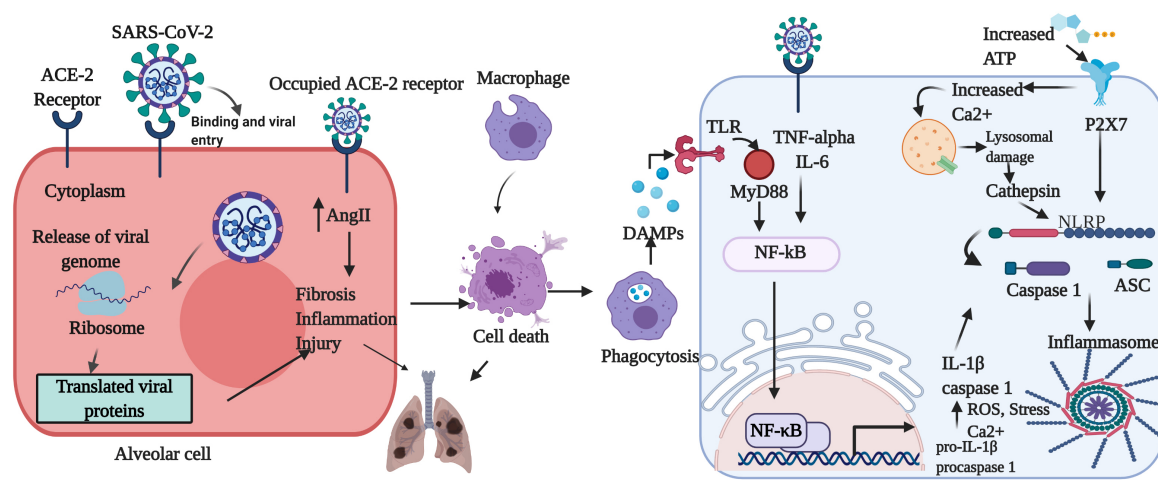

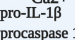

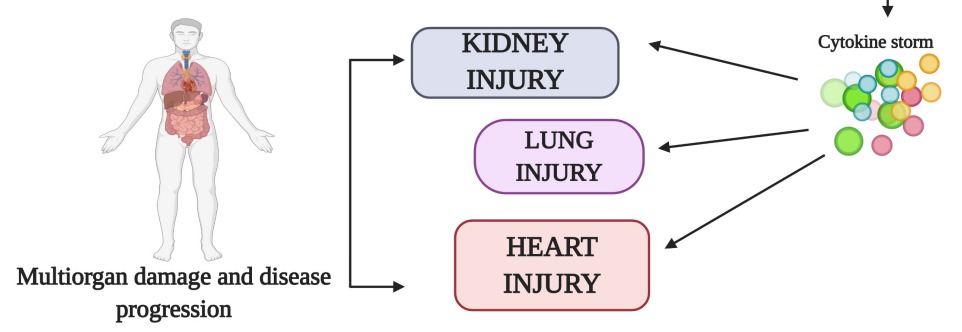

Fig. 1. Role of cytokine storm in COVID-19. When SARS-CoV-2 binds the cell, the ACE2 receptors become occupied. This increases Angll which results in lung fibrosis, inflammation, and damage. The infected cell also undergoes cell death as a result of the viral infection. Macrophages engulf the dead cells and release DAMPSs, which bind the TLR and activated NF- $\kappa \beta$ by means of MyD88. Activated NF- $\kappa \beta$ binding activates the inflammasome. Binding of the virus to the receptor also upregulates IL-6 and TNF-alpha, further activating NF- $\kappa \beta$. Increase in ATP binds the$\mathrm{P} 2 \mathrm{X} 7$ receptor, which in turn increases $\mathrm{Ca} 2+$, which causes lysosomal damage and further activation of the inflammasome. Continuous activation of the inflammasome produces the cytokine storm, resulting in multiorgan damage. 
Table 1. Commonly used sources of MSCs

\begin{tabular}{|c|c|c|c|c|c|c|}
\hline S. No & Source & Extraction route & $\begin{array}{l}\text { Purity } \\
\text { level }\end{array}$ & $\begin{array}{l}\text { Proliferation } \\
\text { rate }\end{array}$ & $\begin{array}{l}\text { Doubling } \\
\text { time }\end{array}$ & MSCs Marker \\
\hline 1. & Bone Marrow & Bone Marrow Aspiration & High & Lowest & $40 \mathrm{Hrs}$ & Stro-1, CD271, SSEA-4, CD146 \\
\hline 2. & Adipose Tissue & Liposuction, lipectomy & Medium & Higher & 5 days & $\mathrm{CD} 271, \mathrm{CD} 146$ \\
\hline 3. & Dental pulp & Tooth extraction or root canal & Low & High & $30-40 \mathrm{Hrs}$ & Stro-1, SSEA-4, CD146 \\
\hline 4. & Umbilical Cord & After birth from umbilical cord & High & Medium & $30 \mathrm{Hrs}$ & CD146 \\
\hline 5. & Wharton's jelly & After birth from umbilical cord & High & High & $30 \mathrm{Hrs}$ & CD73, CD90, CD105 \\
\hline 6. & Placenta & Obtained after delivery & High & High & $36 \mathrm{Hrs}$ & SSEA-4, CD146 \\
\hline 7. & Synovial Fluid & Synovium or synovial fluid & High & High & 10 days & Stro-1, SSEA-4, CD146 \\
\hline 8. & Endometrium & $\begin{array}{l}\text { Endometrium biopsies or } \\
\text { menstrual blood }\end{array}$ & High & High & $18-36 \mathrm{Hrs}$ & Stro-1, CD146 \\
\hline 9. & Peripheral Blood & Density Gradient Centrifugation & Low & Low & $95 \mathrm{Hrs}$ & CD133 \\
\hline
\end{tabular}

adaptive immune system. The direct or indirect interactions of MSCs with the immune cells make the MSCs activate the immunomodulatory responses (19). The immunomodulatory functions of MSCs depend on the environment of the host cells; based on the inflammatory status, the MSCs decide the type of immunoregulatory effect (20). MSCs represent pro-inflammatory immune reactions and anti-inflammatory reactions (21). MSCs regulate the immune system via the transforming growth factor $\beta 1$ (TGF $\beta 1$ ), which can trigger the proliferation of Tregs, induce IL-6, which prevents the proliferation of neutrophils, and stimulate the prostaglandin E2 (PGE2), which inhibits the antigen presentation by dendritic cells and proliferations of T-effector cells $(22,23)$. MSCs mediate these kinds of effects by direct contact, where it releases the regulatory cytokines, such as IFN- $\gamma$, indoleamine 2,3-dioxygenase, TGF $\beta$, IL-10, and PGE2 (24). Moreover, MSCs can hinder the proliferation and/or functions of the CD4+ Th1 and TH17 cells, CD8 + T cells, and the natural killer (NK) cells, mainly by secreting soluble factors, such as TGF $\beta 1$ and hepatocyte growth factor (HGF) (16).

\section{MESENCHYMAL STEM CELLS (MSCs) AND MSC SECRETOME}

It has currently become apparent that MSCs induce therapeutic characteristics by a paracrine pathway by releasing bioactive substances known as secretomes (25). MSC-secretomes are made of soluble proteins, including cytokines, chemokines, growth factors, and extracellular vesicles (EVs), which include microvesicles and exosomes (26). Stem cells release these secretomes by common secretory mechanisms. When the culture medium or secretome are injected into the patients, the neighboring cells assimilate the molecules by paracrine signalling (27). The exosomes themselves contain numerous bioactive molecules, which include microRNAs (miRNA), transfer RNAs (tRNA), long noncoding RNAs (IncRNA), growth factors, proteins, and lipids. The lipid content of the exosomes provide an added advantage by aiding in the infusion of the exosomes with the plasma membrane of the neighboring cells (28). The molecules

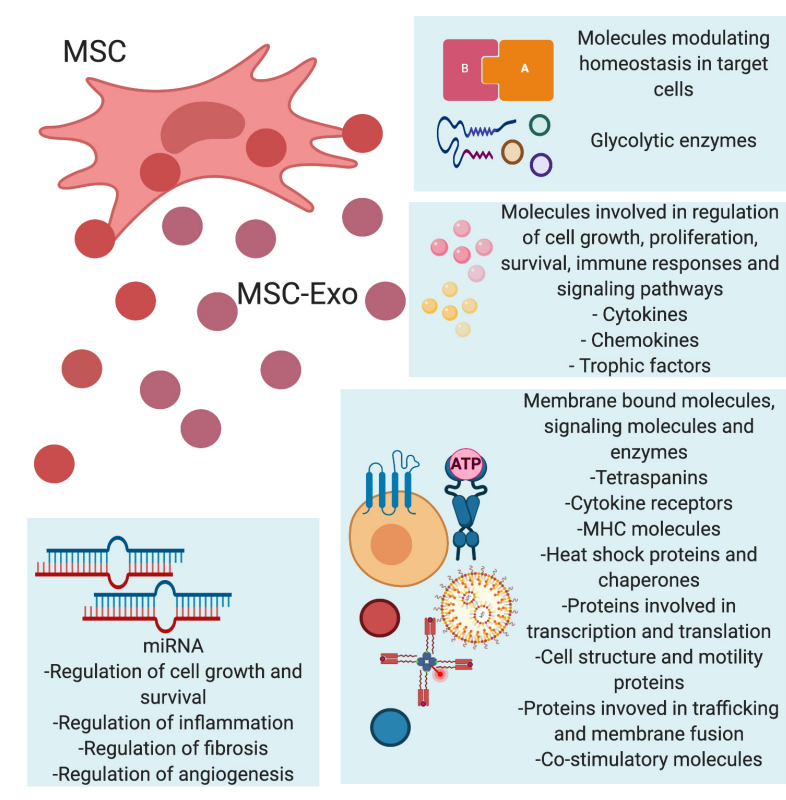

Fig. 2. Molecules released by MSC-Exos. MSC-Exos affect their targets by means of various molecules that they secrete. The MSC-Exos secrete molecules that maintain the homeostasis in the neighboring cells while also secreting glycolytic enzymes. Other molecules involved in cell growth, proliferation, and modulation of the immune response and signalling pathways are secreted by the MSC-Exos. Some membrane-bound molecules that aid in cell signalling and miRNAs with various functions are also released by MSC-Exos.

involved in regulation of cell growth, proliferation, survival, and immune responses are released by exosomes, are elaborately illustrated in Fig. 2. Upon internalization of the molecules in the secretome, the neighboring cells modulate various downstream pathways, including immunomodulation, suppression of apoptosis, prevention of fibrosis, and remodelling of the injured tissues (25). 


\section{IMMUNOMODULATORY POTENTIAL OF MSC-EXOS}

Exosomes are nanoparticles with a diameter of 40-150 nm. To generate and isolate the exosomes, MSCs can be conditioned to increase the release of exosomes by treatment with cytokines or by serum starvation or hypoxia (29). The exosomes are then purified and can be subsequently introduced into the body. MSC-Exos can inhibit CD4 + and CD8 + T cells and NK cells (30). They inhibited T cells expressing IL-17 and induced IL-10-expressing regulatory cells that are involved with suppression of inflammation. MSC-Exos also aid in suppressing the differentiation of $\mathrm{CD} 4+$ and $\mathrm{CD} 8+\mathrm{T}$ cells by releasing molecules like TGF $\beta$ and prevent inflammation in vivo (31). Similarly, treatment with MSC-Exos reduced the proliferation and activation of NK cells (32). MSC-Exos could shift macrophages from the $M 1$ to the M2 phenotype, further suppressing pro-inflammatory states (33). Moreover, sepsis is an important lethal factor in COVID-19 patients, and treatments with MSC-Exos have increased the rate of survival in mice with sepsis (34). Concomitantly, MSC-Exos also suppressed release of the proinflammatory factors TNF- $\alpha$, IFN- $\gamma$, IL-6, IL-17, and IL-1 $\beta$ (35) and promoted release of anti-inflammatory factors, such as IL-4, IL-10 and TGF- $\beta$ (36). Additionally, MSC-Exos also reduced the number of chemokines in the serum when injected (37). These immunomodulatory effects of MSC-Exos have also been attributed to their anti-inflammatory cargo, such as IDO, HLA-G, PD-L1 and galectin-1 $(38,39)$. These mechanisms are illustrated in Fig. 3.

\section{MSC-EXOS THERAPY FOR COVID-19}

In COVID-19, multiorgan damage has been seen in manyinfected individuals. MSC-Exos is known to alleviate lung injury in asthmatic models and ARDS $(40,41)$. MSC-Exos may also be useful in the treatment of cardiovascular (42) and renal problems (43). Hence, they can be used to treat organ damage associated with COVID-19. Similarly, MSC-EVs have also exhibited inhibitory activity on the hemagglutination of avian, swine, and human influenza viruses (44). Likewise, MSC-Exos lowered the death rate in H7N9 patients without any toxic effects during follow-up examinations (45). In addition, these exosomes consist of adhesion molecules that accurately guide them to the injured site. The usage of the exosomes may be preferred to the MSCs, since they can easily cross the bloodbrain barrier, are inexpensive, and cannot undergo independent self-renewal, hence preventing adverse consequences, such as tumor formation. In this pandemic situation, MSC-Exos may be considered as a good treatment option to alleviate the effect of SARS-CoV-2 infection.

\section{CURRENT CLINICAL TRIALS OF STEM CELL-BASED THERAPY IN COVID-19}

Of late, stem-cell-based studies in the treatment of COVID-19

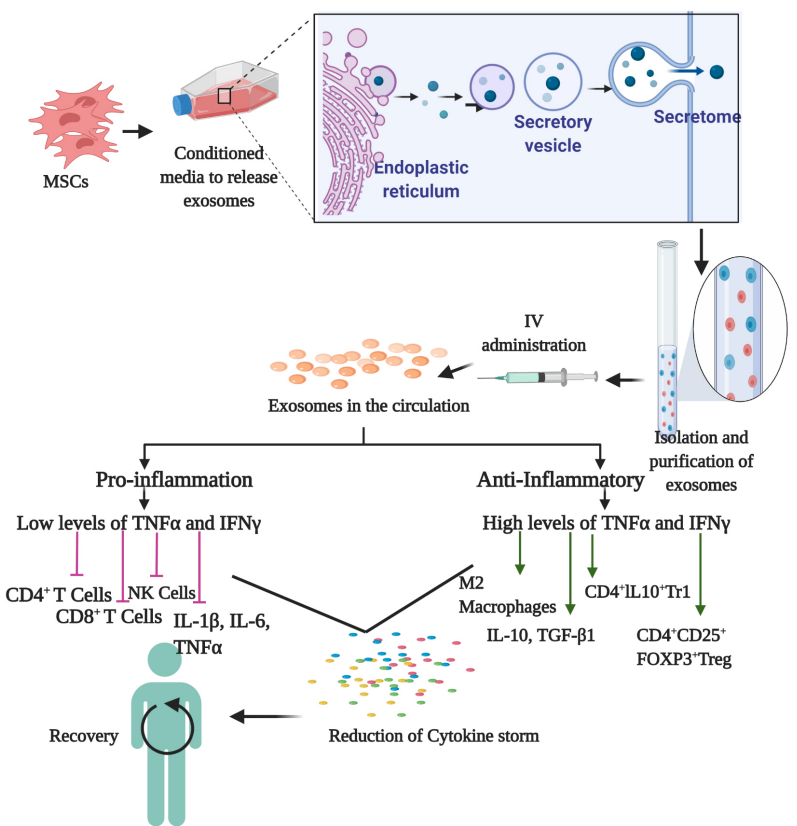

Fig. 3. MSC-Exos therapy for COVID-19. Isolated MSCs are conditioned in specialized media that induce release of exosomes. The MSCs identify the external signal and start to pack regulatory factors in secretory vesicles that are released into the culture medium. The exosomes are identified and isolated using specific markers, and are then administered intravenously the i.v. injection. The exosomes inhibit IL-1, IL-6, NK cells, CD4+, and CD8+. This results in suppression of the cytokine storm. Exosomes also activate IL-10, TGF-beta, M2 macrophages, and T and B regulatory cells to further suppress the immune system. This reduces the proinflammatory cytokines, alleviating symptoms and aiding in recovery of patients.

have been gaining momentum. The efficiency and safety of usage of exosomes that had been obtained from BM-MSCs was recently tested on 24 SARS-CoV-2 patients (46). These patients exhibited moderate to severe ARDS. When the exosomes were introduced into the patients, there were no side effects, and patients improved in clinical status and oxygenation (46). In a similar study, patients treated with MSCs showed a remarkable improvement in pulmonary function, higher levels of peripheral lymphocytes, and a reduction in the cells that trigger the cytokine storm. Interestingly, the MSCs did not exhibit ACE2 or TMPRSS2 expression, showing that they may not be infected with COVID-19 (47). Several clinical trials are in the pipeline for usage of stem cells for the treatment of COVID-19 (Table 2). Wharton's jelly-derived MSCs (WJ-MSCs), which have been used in various studies based on stem-cell therapy and trials, are in progress for their usage for COVID-19 treatment (48). Moreover, adipose tissue-derived AD-MSCs have been used in a few studies in various doses and protocols for COVID-19 therapy (49). Likewise, a novel trial includes inhalation of MSC-Exos for alleviation of symptoms (50). In addition, MSCs from dental pulp (51) and olfactory mucosa 


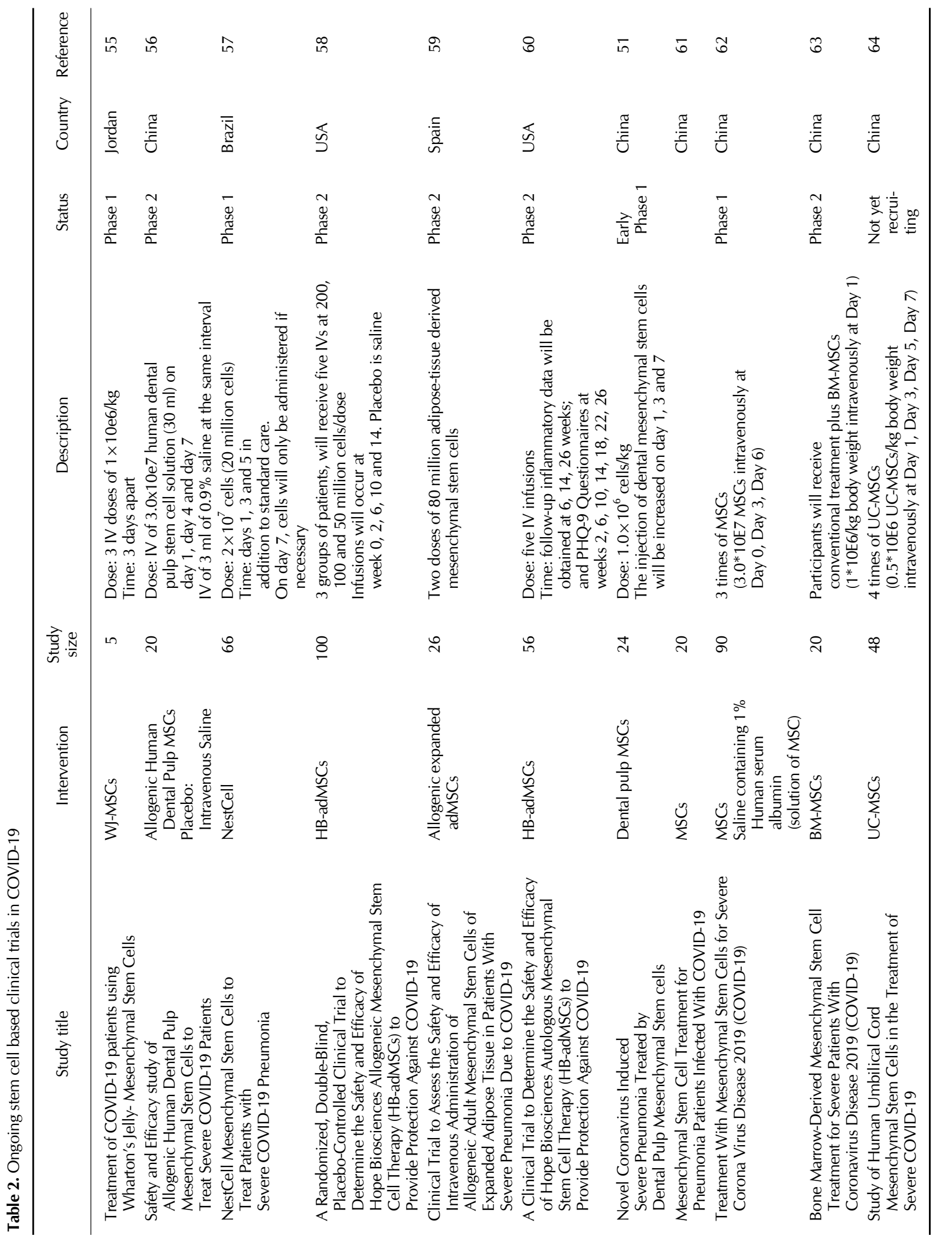




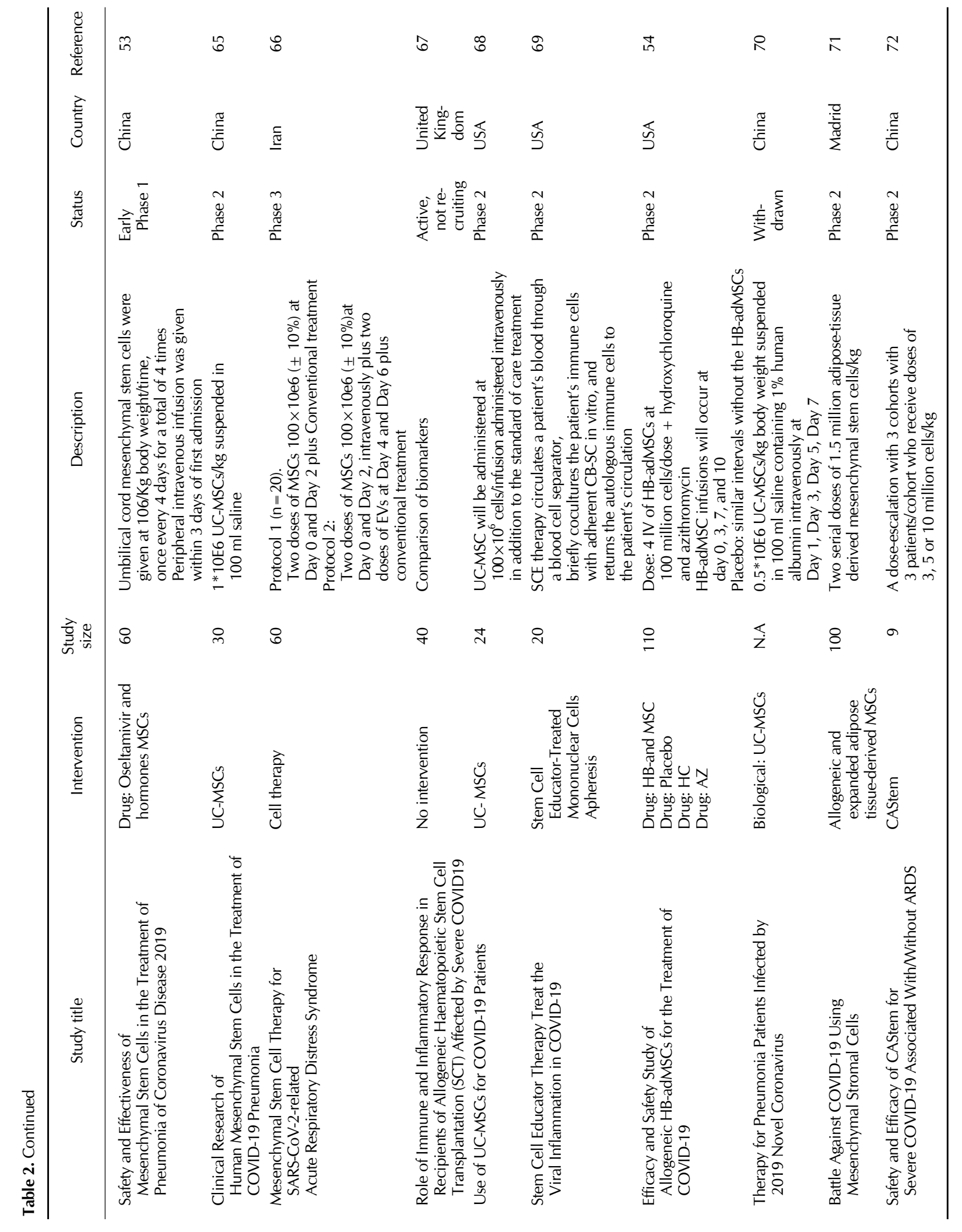




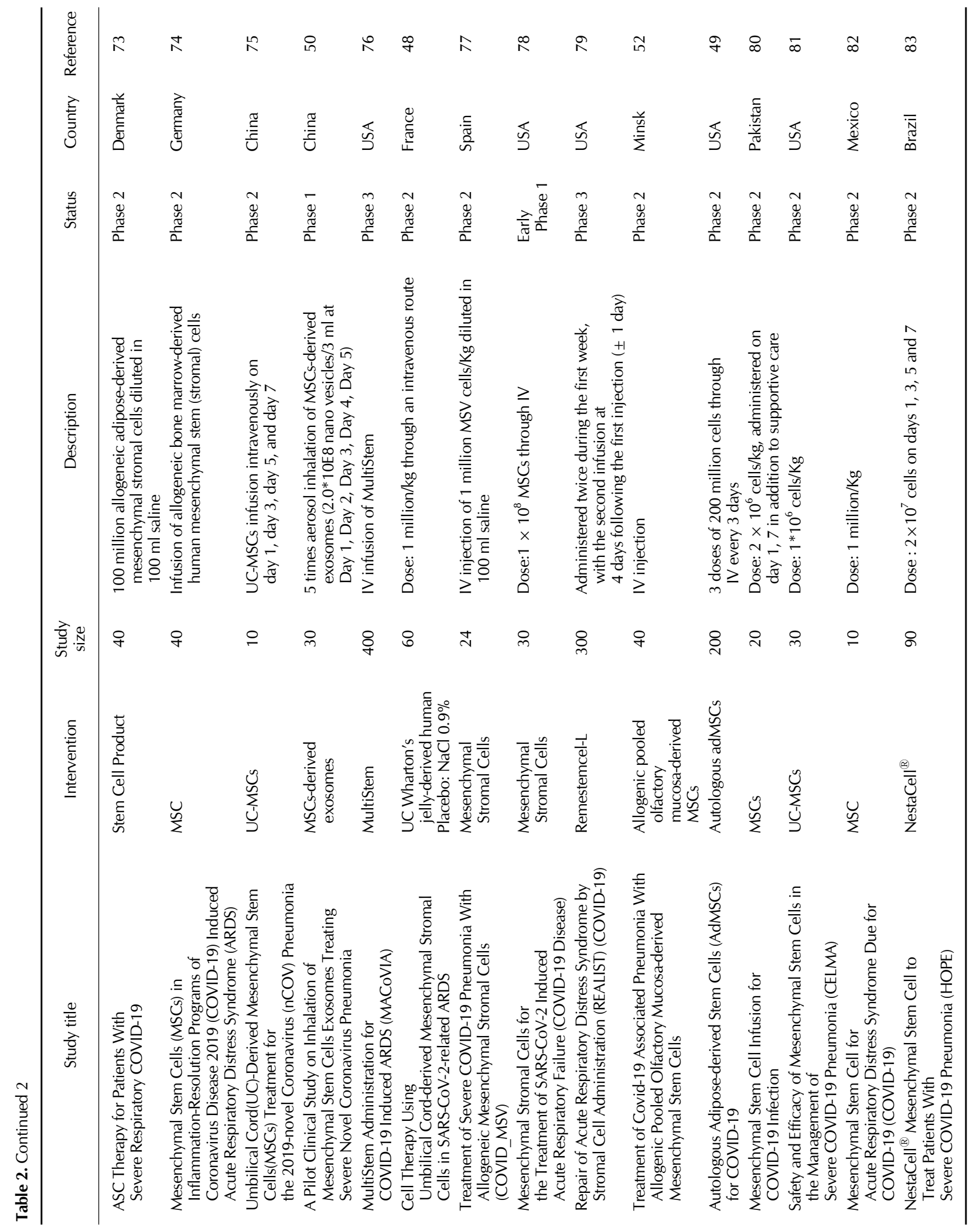




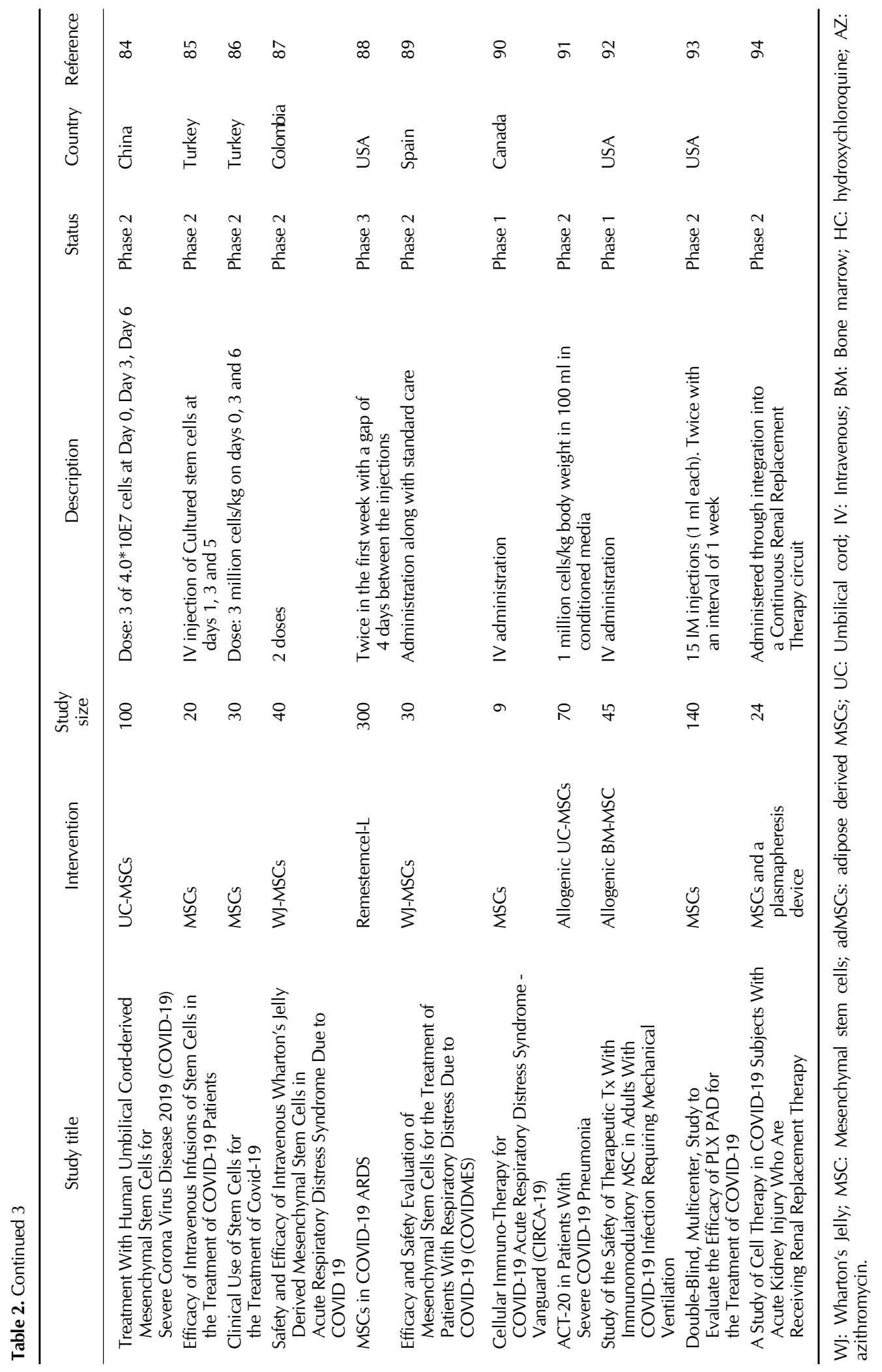


(52) were administered in various doses. MSCs in the clinical trials are predominantly administered intravenously; i.v. injection and, in some studies, MSCs have been given as adjuvant therapy in addition to drugs like oseltamivir, hormones, hydroxychloroquine, and azithromycin (53, 54). These trials reveal promising new routes for the battle against COVID-19 (55-94).

\section{FUTURE DIRECTIONS}

Stem cells have been studied extensively for their ability to regenerate and for the treatment of various diseases. Recently, we devised an improved protocol for the isolation of urinederived stem cells and their further differentiation into immune cells (95). Moreover, our research group promoted the hematopoietic differentiation of hiPSCs using a novel small molecule (96). At the advent of COVID-19, it has become mandatory to discover therapeutic strategies that are easily reproducible and cost effective. Drugs currently available for the treatment of COVID-19 include ones that target viral replication. These drugs include camo-stat mesylate, which is involved in the inhibition of viral fusion to the cell membrane, and favipiravir and remdesivir, which are anti-viral drugs. However, because the cytokine storm is found predominantly in COVID-19 patients, it is essential to consider drugs that inhibit viral replication while treating the cytokine storm. Hence, MSC-Exos may be appropriate therapeutic agents for COVID-19 (97). MSCs can be more advantageous than other anti-inflammatory agents, because they can provide immunomodulatory effects based on the host cells. In addition to these effects, MSCs can prevent fibrosis of tissues, enable reversal of lung dysfunction, and aid in the regeneration of damaged tissue, which can be significantly beneficial for COVID-19-associated organ damage $(98,99)$. Because the healing properties of the MSCs can be primarily attributed to the secretomes or exosomes, using them may be more effective than using MSCs themselves. Exosomes can be mass-produced, administered systematically with minimaltoxicity, and be able to reach the cell targets more efficiently. In addition to their inherent immunomodulatory potential, the MSC-Exos can also be used as a drug-delivery system (100). MSC-Exos can be modified in vivo to release exosomes that have a higher immunomodulatory potential (101) and can be cultured using various cytokines to exhibit an anti-inflammatory state (102). Although MSC-Exos appear to be promising therapeutic agents for COVID-19, more experimental research is necessary for them to be used clinically. Moreover, it is essential to optimize the protocols for storage and isolation of MSC-Exos for the treatment of COVID-19. It is also imperative to do experiments to understand the underlying mechanisms of COVID-19 in order to optimize MSC-Exo therapy for treatment (97). Further, it is also essential to find the optimum dosage, route of administration, and treatment schedule for MSC-Exos. Hence, since MSCs are more widely studied in these aspects than are MSC-Exos, they are predominantly preferred in clinical trials for COVID-19 (103).

\section{CONCLUDING REMARKS}

COVID-19 has invoked frenzy in individuals worldwide. The unceasing increase of infection and death has halted the lives of the citizens of countries everywhere. Hence, it is important to discover novel therapeutic platforms and productive measures without further delay (104). The therapies produced must be easily reproducible and available in large quantities so that enough bioactive molecules will be available for all individuals who have succumbed to COVID-19. MSCs and MSCExos can be used for their immunomodulatory effects in individuals with COVID-19.

\section{ACKNOWLEDGEMENTS}

The author Dr. VB would like to thank Bharathiar University for providing the necessary infrastructure facility and Project funded and supported by MHRD-RUSA 2.0 - BEICH to carry out this manuscript of diagnostic and therapeutic approaches (Ref No. BU/RUSA/BEICH/2019/65).

Dr. SMD would like to thank the Science and Engineering Research Board (SERB) (ECR/2018/000718), Government of India, New Delhi, for providing necessary help in carrying out this review process. The study was supported by a grant from the National Research Foundation (NRF) funded by the Korean government (Grant no: 2019M3A9H1030682).

\section{CONFLICTS OF INTEREST}

The authors have no conflicting interest.

\section{REFERENCES}

1. Vellingiri B, Jayaramayya $K$, Iyer M et al (2020) COVID19: A promising cure for the global panic. Sci Total Environ 725, 138277

2. Iyer M, Jayaramayya K, Subramaniam MD et al (2020) COVID-19: an update on diagnostic and therapeutic approaches. BMB Rep 53, 191-205

3. Grasselli G, Zangrillo A, Zanella A et al (2020) Baseline characteristics and outcomes of 1591 patients infected with SARS-CoV-2 admitted to ICUs of the Lombardy region, Italy. JAMA 323, 1574-1581

4. Sun X, Wang T, Cai D et al (2020) Cytokine storm intervention in the early stages of COVID-19 pneumonia. Cytokine Growth Factor Rev. doi: 10.1016/j.cytogfr.2020. 04.002

5. Balachandar V, Mahalaxmi I, Devi SM et al (2020) Follow-up studies in COVID-19 recovered patients-is it mandatory? Sci Total Environ 729, 139021

6. Taghavi-farahabadi, M, Mahmoudi M, Soudi S and Hashemi SM (2020) Hypothesis for the management and treatment of the COVID-19-induced acute respiratory distress syndrome and lung injury using mesenchymal stem cell- 
derived exosomes. Med Hypotheses 144, 109865

7. Li G, Fan Y, Lai Y et al (2020) Coronavirus infections and immune responses. J Med Virol 92, 424-432

8. Qin C, Zhou L, Hu Z et al (2020) Dysregulation of immune response in patients with COVID-19 in Wuhan, China. Clin Infect Dis. doi: 10.1093/cid/ciaa248

9. Wang W, He J and Wu S (2020) The definition and risks of cytokine release syndrome-like in 11 COVID-19-infected pneumonia critically ill patients: disease characteristics and retrospective analysis. doi: https://doi.org/10.1101/ 2020.02.26.20026989

10. Tufan A, Güler AA and Matucci-Cerinic M (2020) COVID19 , immune system response, hyperinflammation and repurposing antirheumatic drugs. Turk J Med Sci 50, 620632

11. Sakaguchi S, Miyara $M$, Costantino $C M$ and Hafler DA (2010) FOXP3 + regulatory T cells in the human immune system. Nat Rev Immunol 10, 490-500

12. Zbinden-Foncea $H$, Francaux $M$, Deldicque $L$ and Hawley JA (2020) Does high cardiorespiratory fitness confer some protection against pro-inflammatory responses after infection by SARS-CoV-2? Obesity. doi: 10.1002/oby.22849

13. Shneider A, Kudriavtsev A and Vakhrusheva A (2020) Can melatonin reduce the severity of COVID-19 pandemic? Int Rev Immunol 39, 153-162

14. Guo J, Huang Z, Lin L and Lv J (2020) Coronavirus Disease 2019 (COVID-19) and Cardiovascular Disease: A viewpoint on the potential influence of angiotensin-converting enzyme inhibitors/angiotensin receptor blockers on onset and severity of severe acute respiratory syndrome coronavirus 2 infection. J Am Heart Assoc 9, e01 6219

15. Conti $\mathrm{P}$, Ronconi G, Caraffa A et al (2020) Induction of pro-inflammatory cytokines (IL-1 and IL-6) and lung inflammation by Coronavirus-19 (COVI-19 or SARS-CoV-2): anti-inflammatory strategies. J Biol Regul Homeost Agents 34,1

16. Zhao Q, Ren H and Han Z (2016) Mesenchymal stem cells: Immunomodulatory capability and clinical potential in immune diseases. J Cell Immunother 2, 3-20

17. Prockop DJ (2017) The exciting prospects of new therapies with mesenchymal stromal cells. Cytotherapy 19, 18

18. Wilson JG, Liu KD, Zhuo $\mathrm{H}$ et al (2015) Mesenchymal stem (stromal) cells for treatment of ARDS: a phase 1 clinical trial. Lancet Respir Med 3, 24-32

19. Uccelli A, Moretta L and Pistoia V (2008) Mesenchymal stem cells in health and disease. Nat Rev Immunol 8, 726-736

20. Ren G, Zhang L, Zhao X et al (2008) Mesenchymal stem cell-mediated immunosuppression occurs via concerted action of chemokines and nitric oxide. Cell Stem Cell 2, $141-150$

21. Waterman RS, Tomchuck SL, Henkle SL and Betancourt AM (2010) A new mesenchymal stem cell (MSC) paradigm: polarization into a pro-inflammatory MSC1 or an Immunosuppressive MSC2 phenotype. PLoS One 5, e10088

22. Patel SA, Meyer JR, Greco SJ, Corcoran KE and Bryan M, Rameshwar P (2010) Mesenchymal stem cells protect breast cancer cells through regulatory $\mathrm{T}$ cells: role of mesenchymal stem cell-derived TGF- $\beta$. J Immunol 184, 58855894

23. Raffaghello L, Bianchi G, Bertolotto M et al (2008) Human mesenchymal stem cells inhibit neutrophil apoptosis: a model for neutrophil preservation in the bone marrow niche. Stem Cells 26, 151-162

24. Thanunchai $M$, Hongeng $S$ and Thitithanyanont A (2015) Mesenchymal stromal cells and viral infection. Stem Cells Int 2015, 860950

25. Bari E, Ferrarotti I, Saracino L, Perteghella S, Torre ML and Corsico AG (2020) Mesenchymal stromal cell secretome for severe COVID-19 infections: Premises for the therapeutic use. Cells 9, 924

26. Crivelli B, Chlapanidas T, Perteghella S et al (2017) Mesenchymal stem/stromal cell extracellular vesicles: From active principle to next generation drug delivery system. J Controlled Release 262, 104-117

27. Deffune E, Prudenciatti A and Moroz A (2020) Mesenchymal stem cell (MSc) secretome: a possible therapeutic strategy for intensive-care COVID-19 patients. Med Hypotheses 142, 109769

28. Keshtkar S, Azarpira N and Ghahremani MH (2018) Mesenchymal stem cell-derived extracellular vesicles: novel frontiers in regenerative medicine. Stem Cell Res Ther 9, 63

29. Lou G, Chen Z, Zheng M and Liu Y (2017) Mesenchymal stem cell-derived exosomes as a new therapeutic strategy for liver diseases. Exp Mol Med 49, e346-e346

30. Lai P, Chen X, Guo L et al (2018) A potent immunomodulatory role of exosomes derived from mesenchymal stromal cells in preventing CGVHD. J Hematol Oncol 11,135

31. Álvarez V, Sánchez-Margallo FM, Macías-García B et al (2018) The immunomodulatory activity of extracellular vesicles derived from endometrial mesenchymal stem cells on CD4 + T cells is partially mediated by TGFbeta. J Tissue Eng Regen Med 12, 2088-2098

32. Fan $Y$, Herr F, Vernochet A, Mennesson B, Oberlin E and Durrbach A (2019) Human fetal liver mesenchymal stem cell-derived exosome impair natural killer cell function. Stem Cells Dev 28, 44-55

33. Domenis R, Cifù A, Quaglia $S$ et al (2018) Pro inflammatory stimuli enhance the immunosuppressive functions of adipose mesenchymal stem cells-derived exosomes. Sci Rep 8, 13325

34. Song Y, Dou H, Li X et al (2017) Exosomal miR-146a contributes to the enhanced therapeutic efficacy of interleukin-1 $\beta$-primed mesenchymal stem cells against sepsis. Stem Cells 35, 1208-1221

35. Chen W, Huang Y, Han J et al (2016) Immunomodulatory effects of mesenchymal stromal cells-derived exosome. Immunol Res 64, 831-840

36. Ji L, Bao L, Gu Z et al (2019) Comparison of immunomodulatory properties of exosomes derived from bone marrow mesenchymal stem cells and dental pulp stem cells. Immunol Res 67, 432-442

37. He Ping (2019) AB0291E the effect of human umbilical cord mesenchymal stem cells-derived exosomes on chemokines in collagen-induced arthritis rats. BMJ 1606-1606

38. Mardpour S, Hamidieh AA, Taleahmad S, Sharifzad F, 
Taghikhani A and Baharvand $\mathrm{H}$ (2019) Interaction between mesenchymal stromal cell-derived extracellular vesicles and immune cells by distinct protein content. J Cellular Physiol 234, 8249-8258

39. Baharlooi H, Azimi M, Salehi Z and Izad M (2020) Mesenchymal stem cell-derived exosomes: A Promising therapeutic ace card to address autoimmune diseases. Int J Stem Cells 13, 13-23

40. Du Y, Zhuansun Y, Chen R, Lin L, Lin Y and Li J (2018) Mesenchymal stem cell exosomes promote immunosuppression of regulatory $\mathrm{T}$ cells in asthma. Exp Cell Res 363, 114-120

41. Fujita $Y$, Kosaka N, Araya J, Kuwano $K$ and Ochiya $T$ (2015) Extracellular vesicles in lung microenvironment and pathogenesis. Trends Mol Med 21, 533-542

42. Barani B, Rajasingh S and Rajasingh J (2017) Exosomes: Outlook for future cell-free cardiovascular disease therapy. Adv Exp Med Biol 2017, 285-307

43. Tsuji K, Kitamura S and Wada J (2018) Secretomes from mesenchymal stem cells against acute kidney injury: possible heterogeneity. Stem Cells Int 2018, 8693137

44. Khatri M, Richardson LA and Meulia T (2018) Mesenchymal stem cell-derived extracellular vesicles attenuate influenza virus-induced acute lung injury in a pig model. Stem Cell Res Ther 9, 17

45. Chen J, Hu C, Chen L et al (2020) Clinical study of mesenchymal stem cell treating acute respiratory distress syndrome induced by epidemic Influenza A (H7N9) infection, a hint for COVID-19 treatment. Engineering. https://doi.org/10.1016/j.eng.2020.02.006

46. Vikram S, Sascha S, Angel L et al (2020) Exosomes derived from bone marrow mesenchymal stem cells as treatment for severe COVID-19. Stem Cells Dev 29, 747754

47. Leng Z, Zhu R, Hou W et al (2020) Transplantation of ACE2-mesenchymal stem cells improves the outcome of patients with COVID-19 pneumonia. Aging Dis 11, 216-228

48. Clinicaltrials.gov. Cell Therapy Using Umbilical Cordderived Mesenchymal Stromal Cells in SARS-CoV-2-related ARDS (STROMA-CoV2) ClinicalTrials.gov Identifier: NCT04333368. https://clinicaltrials.gov/ct2/show/NCT043 33368?term $=$ Wharton\& results cond $=$ COVIDcovid -19 \&draw $=2$ \& rank $=2$.term $=$ stem + cell \& cntry $=$ \&state $=\&$ city $=\&$ dist $=$. Accessed on 26th June 2020

49. Clinicaltrials.gov. Autologous Adipose-derived Stem Cells (AdMSCs) for COVID-19. ClinicalTrials.gov Identifier: NCT04428801. https://clinicaltrials.gov/ct2/show/NCT0442 8801. Accessed on 26th June 2020

50. Clinicaltrials.gov. A Pilot Clinical Study on Inhalation of Mesenchymal Stem Cells Exosomes Treating Severe Novel Coronavirus Pneumonia. ClinicalTrials.gov Identifier: NCT 04276987.https://clinicaltrials.gov/ct2/show/NCT04276 987. Accessed on 26th June 2020. Accessed on 26th June 2020

51. Clinicaltrials.gov. Novel Coronavirus Induced Severe Pneumonia Treated by Dental Pulp Mesenchymal Stem Cells. ClinicalTrials.gov Identifier: NCT04302519. https:// clinicaltrials.gov/ct2/show/NCT04302519. Accessed on 26th June 2020

52. Clinicaltrials.gov. Treatment of Covid-19 Associated Pneu- monia with Allogenic Pooled Olfactory Mucosa-derived Mesenchymal Stem Cells. ClinicalTrials.gov Identifier: NCT04382547. https://clinicaltrials.gov/ct2/show/NCT043 82547. Accessed on 26th June 2020

53. Clinicaltrials.gov. Safety and Effectiveness of Mesenchymal Stem Cells in the Treatment of Pneumonia of Coronavirus Disease 2019. ClinicalTrials.gov Identifier: NCT04 371601.https://clinicaltrials.gov/ct2/show/NCT0437160 1. Accessed on 26th June 2020

54. Clinicaltrials.gov. Efficacy and Safety Study of Allogeneic HB-adMSCs for the Treatment of COVID-19. ClinicalTrials. gov Identifier: NCT04362189. https://clinicaltrials.gov/ct2/ show/NCT04362189. Accessed on 26th June 2020

55. Clinicaltrials.gov. Treatment of COVID-19 patients using Wharton's Jelly-Mesenchymal Stem Cells. ClinicalTrials. gov Identifier: NCT04313322. https://clinicaltrials.gov/ct2/ show/NCT04313322. Accessed on 16th July 2020

56. Clinicaltrials.gov. Safety and Efficacy study of Allogenic Human Dental Pulp Mesenchymal Stem Cells to Treat Severe COVID-19 Patients. ClinicalTrials.gov Identifier: NCT04336254. https://clinicaltrials.gov/ct2/show/NCT04 336254 . Accessed on 16th July 2020. Accessed on 16th July 2020

57. Clinicaltrials.gov. NestCell Mesenchymal Stem Cells to Treat Patients with Severe COVID-19 Pneumonia. ClinicalTrials.gov Identifier: NCT04315987. https://clinicaltrials. gov/ct2/show/NCT04315987. Accessed on 16th July 2020

58. Clinicaltrials.gov. A Randomized, Double-Blind, PlaceboControlled Clinical Trial to Determine the Safety and Efficacy of Hope Biosciences Allogeneic Mesenchymal Stem Cell Therapy (HB-adMSCs) to Provide Protection Against COVID-19. ClinicalTrials.gov Identifier: NCT04 348435. https://clinicaltrials.gov/ct2/show/NCT04348435. Accessed on 16th July 2020

59. Clinicaltrials.gov. Clinical Trial to Assess the Safety and Efficacy of Intravenous Administration of Allogeneic Adult Mesenchymal Stem Cells of Expanded Adipose Tissue in Patients With Severe Pneumonia Due to COVID-19. ClinicalTrials.gov Identifier: NCT04366323. https://clinicaltrials. gov/ct2/show/NCT04366323. Accessed on 16th July 2020

60. Clinicaltrials.gov. A Clinical Trial to Determine the Safety and Efficacy of Hope Biosciences Autologous Mesenchymal Stem Cell Therapy (HB-adMSCs) to Provide Protection Against COVID-19. ClinicalTrials.gov Identifier: NCT04 349631. https://clinicaltrials.gov/ct2/show/NCT04349631. Accessed on 16th July 2020

61. Clinicaltrials.gov. Mesenchymal Stem Cell Treatment for Pneumonia Patients Infected With COVID-19. ClinicalTrials.gov Identifier: NCT04252118. https://clinicaltrials. gov/ct2/show/NCT04252118. Accessed on 16th July 2020

62. Clinicaltrials.gov. Treatment With Mesenchymal Stem Cells for Severe Corona Virus Disease 2019 (COVID-19) ClinicalTrials.gov Identifier: NCT04288102https://clinical trials.gov/ct2/show/NCT04288102?term $=$ Treatment $+\mathrm{W}$ ith + Mesenchymal + Stem + Cells + + for + Severe + Coron $\mathrm{a}+$ Virus + Disease + 2019+\%28COVID-19\%29\&draw = $2 \&$ rank $=1$. Accessed on 16 th July 2020

63. Clinicaltrials.gov. Bone Marrow-Derived Mesenchymal Stem Cell Treatment for Severe Patients With Coronavirus Disease 2019 (COVID-19) ClinicalTrials.gov Identifier: 
NCT04346368. https://clinicaltrials.gov/ct2/show/NCT04 346368. Accessed on 16th July 2020

64. Clinicaltrials.gov. Study of Human Umbilical Cord Mesenchymal Stem Cells in the Treatment of Severe COVID19. ClinicalTrials.gov Identifier: NCT04273646. https:// clinicaltrials.gov/ct2/show/NCT04273646. Accessed on 16th July 2020

65. Clinicaltrials.gov. Clinical Research of Human Mesenchymal Stem Cells in the Treatment of COVID-19 Pneumonia. ClinicalTrials.gov Identifier: NCT04339660. https:// clinicaltrials.gov/ct2/show/NCT04339660. Accessed on 16th July 2020

66. Clinicaltrials.gov. Mesenchymal Stem Cell Therapy for SARS-CoV-2-related Acute Respiratory Distress Syndrome. ClinicalTrials.gov Identifier: NCT04366063. https://clinical trials.gov/ct2/show/NCT04366063. Accessed on 16th July 2020

67. Clinicaltrials.gov. Role of Immune and Inflammatory Response in Recipients of Allogeneic Haematopoietic Stem Cell Transplantation (SCT) Affected by Severe COVID19. ClinicalTrials.gov Identifier: NCT04349540. https://clini caltrials.gov/ct2/show/NCT04349540. Accessed on 16th July 2020

68. Clinicaltrials.gov. Use of UC-MSCs for COVID-19 Patients. ClinicalTrials.gov Identifier: NCT04355728. https://clini caltrials.gov/ct2/show/NCT04355728. Accessed on 16th July 2020

69. Clinicaltrials.gov. Stem Cell Educator Therapy Treat the Viral Inflammation in COVID-19. ClinicalTrials.gov Identifier: NCT04299152https://clinicaltrials.gov/ct2/show/NCT04 299152.Accessed on 16th July 2020

70. Clinicaltrials.gov. Therapy for Pneumonia Patients Infected by 2019 Novel Coronavirus ClinicalTrials.gov Identifier: NCT04293692. https://clinicaltrials.gov/ct2/show/NCT04 293692. Accessed on 16th July 2020

71. Clinicaltrials.gov. Battle Against COVID-19 Using Mesenchymal Stromal Cells. ClinicalTrials.gov Identifier: NCT 04348461. https://clinicaltrials.gov/ct2/show/NCT04348461. Accessed on 16th July 2020

72. Clinicaltrials.gov. Safety and Efficacy of CAStem for Severe COVID-19 Associated With/Without ARDS ClinicalTrials.gov Identifier: NCT04331613. https://clinicaltrials. gov/ct2/show/NCT04331613. Accessed on 16th July 2020

73. Clinicaltrials.gov. ASC Therapy for Patients With Severe Respiratory COVID-19. ClinicalTrials.gov Identifier: NCT 04341610. https://clinicaltrials.gov/ct2/show/NCT04341610. Accessed on 16th July 2020

74. Clinicaltrials.gov. Mesenchymal Stem Cells (MSCs) in Inflammation-Resolution Programs of Coronavirus Disease 2019 (COVID-19) Induced Acute Respiratory Distress Syndrome (ARDS). ClinicalTrials.gov Identifier: NCT043 77334. https://clinicaltrials.gov/ct2/show/NCT04377334. Accessed on 16th July 2020

75. Clinicaltrials.gov. Umbilical Cord(UC)-Derived Mesenchymal Stem Cells(MSCs) Treatment for the 2019-novel Coronavirus (nCOV) Pneumonia. ClinicalTrials.gov Identifier: NCT04269525. https://clinicaltrials.gov/ct2/show/NCT 04269525. Accessed on 16th July 2020

76. Clinicaltrials.gov. MultiStem Administration for COVID-19 Induced ARDS (MACoVIA) ClinicalTrials.gov Identifier:
NCT04367077. https://clinicaltrials.gov/ct2/show/NCT04 367077 . Accessed on 16th July 2020

77. Clinicaltrials.gov. Treatment of Severe COVID-19 Pneumonia with Allogeneic Mesenchymal Stromal Cells (COVID_MSV) Clinical Trials.gov Identifier: NCT04361 942. https://clinicaltrials.gov/ct2/show/NCT04361942. Accessed on 16th July 2020

78. Clinicaltrials.gov. Mesenchymal Stromal Cells for the Treatment of SARS-CoV-2 Induced Acute Respiratory Failure (COVID-19 Disease). ClinicalTrials.gov Identifier: NCT04345601. https://clinicaltrials.gov/ct2/show/NCT04 345601. Accessed on 16th July 2020

79. Clinicaltrials.gov. Repair of Acute Respiratory Distress Syndrome by Stromal Cell Administration (REALIST) (COVID19)ClinicalTrials.gov Identifier: NCT03042143. https://clini caltrials.gov/ct2/show/NCT03042143. Accessed on 16th July 2020

80. Clinicaltrials.gov. Mesenchymal Stem Cell Infusion for COVID-19 Infection. ClinicalTrials.gov Identifier: NCT 04444271. https://clinicaltrials.gov/ct2/show/NCT044442 71. Accessed on 16 th July 2020

81. Clinicaltrials.gov. Safety and Efficacy of Mesenchymal Stem Cells in the Management of Severe COVID-19 Pneumonia (CELMA). ClinicalTrials.gov Identifier: NCT 04429763. https://clinicaltrials.gov/ct2/show/NCT044297 63. Accessed on 16th July 2020

82. Clinicaltrials.gov. Mesenchymal Stem Cell for Acute Respiratory Distress Syndrome Due for COVID-19 (COVID19). ClinicalTrials.gov Identifier: NCT04416139. https:// clinicaltrials.gov/ct2/show/NCT04416139. Accessed on 16 th July 2020

83. Clinicaltrials.gov. NestaCell ${ }^{\circledR}$ Mesenchymal Stem Cell to Treat Patients With Severe COVID-19 Pneumonia (HOPE). ClinicalTrials.gov Identifier: NCT04315987. https://clini caltrials.gov/ct2/show/NCT04315987. Accessed on 16th July 2020

84. Clinicaltrials.gov. Treatment With Human Umbilical Cordderived Mesenchymal Stem Cells for Severe Corona Virus Disease 2019 (COVID-19). ClinicalTrials.gov Identifier: NCT04288102. https://clinicaltrials.gov/ct2/show/NCT 04288102 . Accessed on 16th July 2020

85. Clinicaltrials.gov. Efficacy of Intravenous Infusions of Stem Cells in the Treatment of COVID-19 Patients . ClinicalTrials.gov Identifier: NCT04437823. https://clinicaltri als.gov/ct2/show/NCT04437823. Accessed on 16th July 2020

86. Clinicaltrials.gov. Clinical Use of Stem Cells for the Treatment of Covid-19. ClinicalTrials.gov Identifier: NCT 04392778. https://clinicaltrials.gov/ct2/show/NCT04392 778. Accessed on 16th July 2020

87. Clinicaltrials.gov. Safety and Efficacy of Intravenous Wharton's Jelly Derived Mesenchymal Stem Cells in Acute Respiratory Distress Syndrome Due to COVID 19. ClinicalTrials.gov Identifier: NCT04390152. https://clini caltrials.gov/ct2/show/NCT04390152. Accessed on 16th July 2020

88. Clinicaltrials.gov. MSCs in COVID-19 ARDS .Clinical Trials.gov Identifier: NCT04371393 https://clinicaltrials. gov/ct2/show/NCT04371393. Accessed on 16th July 2020

89. Clinicaltrials.gov. Efficacy and Safety Evaluation of Mesen- 
chymal Stem Cells for the Treatment of Patients With Respiratory Distress Due to COVID-19 (COVIDMES). ClinicalTrials.gov Identifier: NCT04390139. https://clini caltrials.gov/ct2/show/NCT04390139. Accessed on 16th July 2020

90. Clinicaltrials.gov. Cellular Immuno-Therapy for COVID19 Acute Respiratory Distress Syndrome - Vanguard (CIRCA-19). ClinicalTrials.gov Identifier: NCT04400032. https://clinicaltrials.gov/ct2/show/NCT04400032. Accessed on 16th July 2020

91. Clinicaltrials.gov. ACT-20 in Patients With Severe COVID19 Pneumonia. ClinicalTrials.gov Identifier: NCT04398303. https://clinicaltrials.gov/ct2/show/NCT04398303. Accessed on 16th July 2020

92. Clinicaltrials.gov. Study of the Safety of Therapeutic Tx With Immunomodulatory MSC in Adults With COVID19 Infection Requiring Mechanical Ventilation ClinicalTrials.gov Identifier: NCT04397796. https://clinicaltrials. gov/ct2/show/NCT04397796. Accessed on 16th July 2020

93. Clinicaltrials.gov. Double-Blind, Multicenter, Study to Evaluate the Efficacy of PLX PAD for the Treatment of COVID-19ClinicalTrials.gov Identifier: NCT04389450. https://clinicaltrials.gov/ct2/show/NCT04389450. Accessed on 16th July 2020

94. Clinicaltrials.gov. A Study of Cell Therapy in COVID-19 Subjects With Acute Kidney Injury Who Are Receiving Renal Replacement Therapy. ClinicalTrials.gov Identifier: NCT04445220 https://clinicaltrials.gov/ct2/show/NCT04 445220. Accessed on 16th July 2020

95. Kim K, Gil M, Dayem AA et al (2020) Improved isolation and culture of urine-derived stem cells (USCs) and enhanced production of immune cells from the USC-derived induced pluripotent stem cells. J Clin Med 9,827
96. Kim K, Abdal Dayem A, Gil M et al (2020) 3,2'Dihydroxyflavone Improves the proliferation and survival of human pluripotent stem cells and their differentiation into hematopoietic progenitor cells. J Clin Med 9, 669

97. Tsuchiya A, Takeuchi S, Iwasawa T et al (2020) Therapeutic potential of mesenchymal stem cells and their exosomes in severe novel coronavirus disease 2019 (COVID-19) cases. Inflammation and Regeneration 40, $1-6$

98. Akkoc T (2020) COVID-19 and mesenchymal stem cell treatment; mystery or not. Adv Exp Med Biol. 1-10 https:// doi.org/10.1007/5584 2020557

99. Pinky, Gupta S, Krishnakumar V et al (2020) Mesenchymal stem cell derived exosomes: a nano platform for therapeutics and drug delivery in combating COVID-19. Stem Cell Rev Rep 1-11 doi: 10.1007/s12015-020-10002-z

100. Muraca M, Pessina A, Pozzobon M et al (2020) Mesenchymal stromal cells and their secreted extracellular vesicles as therapeutic tools for COVID-19 pneumonia?. J Control Release 325, 135-140

101. O'Driscoll L (2020) Extracellular vesicles from mesenchymal stem cells as a Covid-19 treatment. Drug Discovery Today. S1359-6446(20)30170-7. https://doi.org/ 10.1016/j.drudis.2020.04.022

102. Sleem A and Saleh F (2020) Mesenchymal stem cells in the fight against viruses: Face to face with the invisible enemy. Curr Res Transl Med S2452-3186(20)30031-3. doi: 10.1016/j.retram.2020.04.003

103. Harrell CR, Jovicic N, Djonov V et al (2020) Therapeutic Use of Mesenchymal Stem Cell-Derived Exosomes: From Basic Science to Clinics. Pharmaceutics 12, 474

104. Balachandar V, Mahalaxmi I, Kaavya J et al (2020) COVID-19: emerging protective measures. Eur Rev Med Pharmacol Sci 24, 3422-3425 